Pacific Journal of Mathematics

QUASITRIANGULAR OPERATOR ALGEBRAS 


\title{
QUASITRIANGULAR OPERATOR ALGEBRAS
}

\author{
JoAn K. Plastiras
}

Fix a sequence $\mathscr{P}=\left\{P_{n}\right\}_{n=1}^{\infty}$ of finite dimensional projections increasing to the identity on a separable Hilbert space $\mathscr{H}$ and let $\mathscr{L}(\mathscr{H})$ denote the algebra of all bounded operators on $\mathscr{H}$. The quasitriangular algebra associated with $\mathscr{P}$ and denoted as $\mathscr{2} \mathcal{T}(\mathscr{P})$ is defined to be the set of those operators $T$ in $\mathscr{L}(\mathscr{H})$ for which $\left\|P_{n}^{\perp} T P_{n}\right\| \rightarrow 0$.

In this paper we will examine the structure of the $\mathscr{Q T}(\mathscr{P})$ algebras. Specifically, if $\mathscr{R}=\left\{R_{n}\right\}_{n=1}^{\infty}$ is another sequence of finite dimensional projections increasing to the identity on the same Hilbert space, when is $\mathscr{2} \mathscr{T}(\mathscr{R})$ equal to $\mathscr{2} \mathscr{T}(\mathscr{P})$ ? By an algebraic isomorphism between two algebras we shall mean a bijection which preserves algebraic structure: that is to say - addition, scalar multiplication, multiplication, but we do not impose any topological condition. When are two quasitriangular algebras isomorphic?

In [5] we asked the same questions of $\mathscr{D}(\mathscr{E})+\mathscr{C}(\mathscr{H})=\{T+K: T$ belongs to the commutant of $E$ and $K$ is compact $\}$ and answered them completely by arguments very different from those presented here; the conclusions were different too. The concept of quasitriangularity for operators was first isolated for systematic study in [3]. The quasitriangular algebra was introduced later in [1] and a formula expressing the distance from such an algebra to an arbitrary operator was obtained. We begin our discussion with an algebraic property:

Definition 1. A subset $\mathscr{S}$ of $\mathscr{L}(\mathscr{H})$ is said to be inverse-closed if whenever $T$ in $\mathscr{S}$ is invertible in $\mathscr{L}(\mathscr{H})$ then $T^{-1}$ belongs to $\mathscr{S}$.

Lemma 2. $2 \mathscr{T}(\mathscr{P})$ is inverse-closed for every sequence $\mathscr{P}=\left\{P_{n}\right\}_{n=1}^{\infty}$ of finite dimensional projections increasing to the identity on a Hilbert space.

Before verifying Lemma 2 we remark that the assumption that the $P_{k}$ be finite dimensional is essential.

Proof. From [1, Corollary following 2.2] we know that $\mathscr{2} \mathscr{T}(\mathscr{P})=$ $\mathscr{T}(\mathscr{P})+\mathscr{C}(\mathscr{H})$, where $\mathscr{T}(\mathscr{P})$ is the set of operators $T$ such that $P_{n}^{\perp} T P_{n}=0$ for all $n$. Hence, it suffices to assume that $S$ belongs to $\mathscr{T}(\mathscr{P})+\mathscr{C}(\mathscr{H})$ and is invertible in $\mathscr{L}(\mathscr{H})$ and show that $S^{-1}$ belongs to $\mathscr{2} \mathscr{T}(\mathscr{P})$. So, $S=T+C$, where $T \in \mathscr{T}(\mathscr{P})$ and $C \in \mathscr{C}(\mathscr{H})$. Since $S_{m}=T+P_{m} C P_{m}$ tends in norm to $S, S_{m}$ is invertible for all $m$ greater than a positive 
integer $l$. Fix $m>l$ and note that $S_{m} P_{n}=P_{n} S_{m} P_{n}$ for all $n \geqq m$, and since $\operatorname{dim} P_{n}<\infty, S_{m}$ maps $P_{n} \mathscr{H}$ onto itself, so that $P_{n} S_{m}^{-1} P_{n}=S_{m}^{-1} P_{n}$ (or equivalently, $\left.P_{n}^{\perp} S_{m}^{-1} P_{n} \equiv 0\right)$. Hence, $S_{m}^{-1}$ belongs to $2 \mathscr{T}(\mathscr{P})$ by definition. As $S_{m}^{-1}$ tends in norm to $(T+C)^{-1}$ and $\mathscr{Q T}(\mathscr{P})$ is norm-closed [1, Proposition 2.1], we conclude that $(T+C)^{-1}$ belongs to $2 \mathcal{T}(\mathscr{P})$.

THEOREM 3. Suppose that $T$ is an invertible operator in $\mathscr{L}(\mathscr{H})$. Then $T$ implements an automorphism of $\mathscr{2} \mathscr{T}(\mathscr{P})$ (i.e. $T \mathscr{2} \mathscr{T}(\mathscr{P}) T^{-1}=\mathscr{Q} \mathscr{T}(\mathscr{P})$ ) if and only if $T$ belongs to $\mathscr{Q} \mathscr{T}(\mathscr{P})$.

Proof. $\Leftarrow$ : Assume that $T$ belongs to $\mathscr{2} \mathscr{T}(\mathscr{P})$. To show that $T$ implements an inner automorphism of $2 \mathscr{T}(\mathscr{P})$ it will suffice to show that $T^{-1}$ also belongs to $\mathscr{2} \mathscr{T}(\mathscr{P})$. But that is immediate from Lemma 2.

$\Rightarrow$ : Assume that $T$ implements an automorphism of $\mathscr{Q} \mathscr{T}(\mathscr{P})$. First we conclude from [1, Theorem 3.3] that $T$ admits a factorization $T=U A$, where $A$ belongs to $\mathscr{T}(\mathscr{P})$ and $U$ is a partial isometry. Note that $A=U^{*} T$ has closed range; since ker $A=\{0\}, A$ is semi-Fredholm by definition. Since $A$ belongs to $\mathscr{2} \mathscr{T}(\mathscr{P})$ the index of $A$ is nonnegative [2] so that $\operatorname{ker} A^{*}=\{0\}$ and $A$ is consequently invertible. This forces $U$ to be unitary. Since $A \in \mathscr{2} \mathscr{T}(\mathscr{P})$ is invertible, then by the previous argument, $A$ implements an automorphism of $\mathscr{2} \mathscr{T}(\mathscr{P})$ so that we are reduced to showing that if $U$ is a unitary operator which implements an automorphism of $\mathscr{2} \mathscr{T}(\mathscr{P})$, then $U$ belongs to $2 \mathscr{T}(\mathscr{P})$.

So, we assume that $U$ does not belong to $2 \mathscr{T}(\mathscr{P})$ and arrive at a contradiction. Since $U$ does not belong to $2 \mathscr{T}(\mathscr{P})$ then by the definition of $\mathscr{2} \mathscr{T}(\mathscr{P})$ there is an $\alpha>0$ and a subsequence $\left\{P_{n(k)}\right\}_{k=1}^{\infty}$ of $\mathscr{P}$ for which $\underline{\lim }_{k}\left\|P_{n(k)}^{\perp} U P_{n(k)}\right\| \geqq \alpha$. From Lemma 2 we know that $U^{*}$ does not belong to $\mathscr{2} \mathscr{T}\left(\left\{P_{n(k)}\right\}_{n=1}^{\infty}\right)$, so that by definition, there is $\beta>0$ and a subsequence $\{m(k)\}_{k=1}^{\infty}$ of $\{n(k)\}_{k=1}^{\infty}$ for which $\underline{\lim }_{k}\left\|P_{m(k)}^{\perp} U^{*} P_{m(k)}\right\| \geqq \beta$. If we let $\epsilon=\min (\alpha, \beta) / 2$, then we can conclude that $\left\|P_{n}^{\perp} U P_{n}\right\|$ and $\left\|P_{n} U P_{n}^{\perp}\right\|$ $\left(=\left\|P_{n}^{\perp} U^{*} P_{n}\right\|\right)$ are both greater than $\epsilon$ for all $n$ in an infinite subset $M$ of N.

We will obtain a sequence $\left\{m_{i}, n_{i}\right\}_{i=1}^{\infty}$ of positive integers such that $0<m_{1}<n_{1}<m_{2}<n_{2}<\cdots$ and projections $\left\{F_{k}, E_{k}\right\}_{k=1}^{\infty}$ such that $F_{k}=$ $P_{m_{k}} P_{n_{k-1}}^{\perp}$ and $E_{k}=P_{n_{k}} P_{m_{k}}^{\perp}$ for which $\left\|F_{k} U E_{k}\right\|$ and $\left\|E_{k} U F_{k}\right\|$ are both greater than $\epsilon / 2$. We do so inductively.

For $k=1$, define $F_{1}=P_{m_{1}}$, where $m_{1}$ is the first integer in $M$. Let $n_{1}$ be the first integer such that $\left\|P_{n_{1}} P_{m_{1}}^{\perp} U P_{m_{1}}\right\|$ and $\left\|P_{m_{1}} U P_{m_{1}}^{\perp} P_{n_{1}}\right\|$ are both greater than $\epsilon / 2$ (such an $n_{1}$ exists because $\left\|P_{m_{1}}^{\perp} U P_{m_{1}}\right\|$ and $\left\|P_{m_{1}} U P_{m_{1}}^{\perp}\right\|$ are greater than $\epsilon$ and the $P_{n}$ tend strongly to the identity).

Assume that we have obtained $\left\{E_{k}, F_{k}\right\}_{k=1}^{l}$. To obtain $m_{l+1}$ and $n_{l+1}$, note that $U P_{n l}$ and $P_{n l} U$ are compact; hence, there is a positive integer $j$ such that $\left\|P_{n}^{\perp} U P_{n l}\right\|$ and $\left\|P_{n_{l}} U P_{n}^{\perp}\right\|$ are both less than $\epsilon / 4$ for all $n \geqq j$. Let $m_{l+1}$ be the first integer in $M$ greater than $j$. 
Then

$$
\begin{aligned}
\left\|P_{m_{l+1}}^{\perp} U P_{m_{l+1}} P_{n_{1}}^{\perp}\right\| & \geqq\left\|P_{m_{l+1}}^{\perp} U P_{m_{l+1}}\right\|-\left\|P_{m_{l+1}}^{\perp} U P_{m_{l+1}} P_{n_{l}}\right\| \\
& \geqq \epsilon-\left\|P_{m_{l+1}}^{\perp} U P_{n_{l}}\right\| \\
& \geqq \epsilon-\epsilon / 4=\frac{3}{4} \epsilon .
\end{aligned}
$$

Similarly, $\left\|P_{m_{l+1}} P_{n_{l}}^{\perp} U P_{m_{l+1}}^{\perp}\right\| \geqq 3 \epsilon / 4$ by the same argument. Let $n_{l+1}$ be the first positive integer greater than $m_{l+1}$ for which $\left\|P_{n_{l+1}} P_{m_{l+1}}^{\perp} U P_{m_{l+1}} P_{n_{l}}^{\perp}\right\|$ and $\left\|P_{m_{l+1}} P_{n_{l}}^{\perp} U P_{m_{l+1}}^{\perp} P_{n_{l+1}}\right\|$ are both greater than $\epsilon / 2$. Let $F_{l+1}=P_{m_{l+1}} P_{n_{l}}^{\perp}$ and let $E_{l+1}=P_{n++1} P_{m_{l+1}}^{\perp}$. Continue inductively.

We select a subsequence $\left\{E_{i}, F_{i,}\right\}_{i=1}^{\infty}$ of $\left\{E_{i}, F_{i}\right\}_{i=1}^{\infty}$ as follows: first, we let $\left\{\alpha_{i j}\right\}_{i, j=1}^{\infty}$ be any sequence of positive real numbers such that $\sum_{i, j} \alpha_{i j}^{2} \leqq$ $\epsilon^{2} / 16$. Let $i_{1}=1$. Assuming that we have obtained $i_{k}$, let $i_{k+1}$ be the next positive integer such that for all $l \supsetneqq k+1,\left\|E_{i_{k+1}} U F_{i_{l}}\right\|$ and $\left\|F_{i_{k+1}} U E_{i_{l}}\right\|$ are less than $\alpha_{k+1, l}$ while $\left\|E_{i l} U F_{i_{k+1}}\right\|$ and $\left\|F_{i l} U E_{i_{k+1}}\right\|$ are less than $\alpha_{l, k+1}$. This is possible because $U F_{i}, F_{i} U$ (respectively $U E_{i,}, E_{i l} U$ ) are compact and the $E_{i}$ (respectively $F_{i}$ ) tend weakly to zero. Continue inductively. Now for each $i_{k}$ there is a rank one partial isometry $T_{i_{k}} \in \mathscr{L}\left(E_{i_{k}} \mathscr{H}, F_{i k} \mathscr{H}\right)$ such that $\left\|E_{i_{k}} U T_{i_{k}} U^{*} F_{i_{k}}\right\| \geqq \epsilon^{2} / 4$. Clearly, $T=\sum_{k=1}^{\infty} T_{i_{k}}$ is a partial isometry in $\mathscr{T}(\mathscr{P})$. So, for arbitrary $l$ in $\mathbf{N}$,

$$
E_{i l}\left(U T U^{*}\right) F_{i l}=\sum_{k=1}^{\infty} E_{i l} U T_{i k} U^{*} F_{i l}=E_{i l} U T_{i l} U^{*} F_{i l}+\sum_{\substack{k=1 \\ k \neq 1}}^{\infty} E_{i l} U T_{i k} U^{*} F_{i,}
$$

Hence,

$$
\begin{aligned}
& \left\|E_{i l}\left(U T U^{*}\right) F_{i l}\right\|+\left\|\sum_{\substack{k=1 \\
k \neq l}}^{\infty} E_{i l} U T_{i k} U^{*} F_{i l}\right\| \geqq\left\|E_{i l} U T_{i l} U^{*} F_{i l}\right\| . \\
& \left\|E_{i l}\left(U T U^{*}\right) F_{i l}\right\|+\sum_{\substack{k=1 \\
k \neq l}}^{\infty}\left\|E_{i l} U T_{i_{k}} U^{*} F_{i l}\right\| \geqq(\epsilon / 2)^{2}=\epsilon^{2} / 4 .
\end{aligned}
$$

Therefore,

$$
\begin{aligned}
\left|E_{i l}\left(U T U^{*}\right) F_{i l}\right| & \geqq \frac{\epsilon^{2}}{4}-\sum_{k \neq l}\left\|E_{i l} U F_{i k}\right\| \cdot\left\|E_{i k} U^{*} F_{i l}\right\| \\
& \geqq \frac{3 \epsilon^{2}}{16} .
\end{aligned}
$$

Since $i_{l}$ was arbitrary, it follows from the construction that

$$
\frac{3 \epsilon^{2}}{16} \leqq\left\|E_{i l}\left(U T U^{*}\right) F_{i l}\right\| \leqq\left\|P_{m_{i}}^{\perp}\left(U T U^{*}\right) P_{m_{i}}\right\|
$$


Hence,

$$
\varlimsup_{k}\left\|P_{k}^{\perp}\left(U T U^{*}\right) P_{k}\right\|>0
$$

and it follows by definition of $\mathscr{Q T}(\mathscr{P})$ that $U T U^{*}$ does not belong to $\mathscr{2} \mathscr{T}(\mathscr{P})$. This contradicts our assumption that $U$ implements an automorphism of $\mathscr{2} \mathscr{T}(\mathscr{P})$ and thus concludes the argument of the proof of Theorem 3.

Definition 4. Let $\mathscr{P}=\left\{P_{n}\right\}_{n=1}^{\infty}$ be a sequence of finite dimensional projections increasing to the identity on a Hilbert space $\mathscr{H}$. An operator $T$ is said to be strictly upper triangular for $\mathscr{P}$ if $P_{n}^{\perp} T P_{n+1}=0$ for all $n$ in $\mathbf{N}$.

REMARK 5. Note that in the proof of Theorem 3 we showed that if $U$ does not belong to $\mathscr{Q} \mathscr{T}(\mathscr{P})$ then there is an operator $T$, which is strictly upper triangular for $\mathscr{P}$, and such that $U T U^{*}$ does not belong to $\mathscr{Q} \mathscr{T}(\mathscr{P})$.

REMARK 6. Let $\mathscr{S}=\left\{S_{n}\right\}_{n=1}^{\infty}$ be any sequence of finite dimensional projections increasing to the identity on $\mathscr{H}$. Let $\mathscr{P}=\left\{P_{n}\right\}_{n=1}^{\infty}$ be a subsequence of $\mathscr{S}$. Then $\mathscr{2} \mathscr{T}(\mathscr{S}) \subseteq 2 \mathscr{T}(\mathscr{P})$. Equality may fail; however, if $T$ is strictly upper triangular for $\mathscr{P}$ then $T$ belongs to $\mathscr{Q} \mathscr{T}(\mathscr{S})$.

DEFINITION 7. A sequence of projections $\mathscr{S}=\left\{S_{n}\right\}_{n=1}^{\infty}$ increasing to the identity on a Hilbert space $\mathscr{H}$ is said to be a defining sequence for a quasitriangular algebra $\mathscr{A}$ if and only if $\mathscr{A}=\left\{T \in \mathscr{L}(\mathscr{H}):\left\|S_{n}^{\perp} T S_{n}\right\| \rightarrow 0\right\}$.

REMARK 8. Suppose that $U$ is a unitary operator which implements an isomorphism $T \rightarrow U T U^{*}$ from $\mathscr{2} \mathscr{T}(\mathscr{P})$ onto $\mathscr{Q T}(\mathscr{T})$. Then $U$ maps defining sequences of $\mathscr{2} \mathscr{T}(\mathscr{P})$ to defining sequences of $\mathscr{2} \mathscr{T}(\mathscr{P})$.

LEMmA 9. Suppose that $\mathscr{P}=\left\{P_{n}\right\}_{n=1}^{\infty}$ and $\mathscr{S}=\left\{S_{n}\right\}_{n=1}^{\infty}$ are sequences of finite dimensional projections increasing to the identity such that $\mathscr{P} \cup \mathscr{S}$ is totally ordered by inclusion. Then $\mathscr{2 T}(\mathscr{P})=\mathscr{2} \mathscr{T}(\mathscr{Y})$ if and only if there exist positive integers $m_{0}$ and $n_{0}$ such that $P_{m_{0}+k}=S_{n_{0}+k}$ for all $k$ in $\mathbf{N}$.

Proof. $\Leftarrow$ : This conclusion is clear.

$\Rightarrow$ : Assume that $\mathscr{2} \mathscr{T}(\mathscr{P})=\mathscr{Q} \mathscr{T}(\mathscr{P})$. Then $\mathscr{2} \mathscr{T}(\mathscr{P})=\mathscr{2} \mathscr{T}(\mathscr{P} \cup \mathscr{S})$. We assert that $\mathscr{P}$ contains all but perhaps finitely many of the projections in $\mathscr{P} \cup \mathscr{S}$. Contrapositively, assume not. Let $\mathscr{R}=\left\{R_{n}\right\}_{n=1}^{\infty}$ be a total ordering of $\mathscr{P} \cup \mathscr{S}$ and choose an infinite subsequence $\left\{n_{k}\right\}_{k=1}^{\infty}$ for which $R_{n_{k}} \notin \mathscr{P}$ but $R_{n_{k}+1} \in \mathscr{P}$. Let $T_{k}$ be any rank one partial isometry with initial space $\left(R_{n k} \ominus R_{n_{k}-1}\right) \mathscr{H}$ and final space $\left(R_{n_{k}+1} \ominus R_{m_{k}}\right) \mathscr{H}$. Then $T=\sum_{k=1}^{\infty} T_{k}$ is a partial isometry which belongs to $\mathscr{2} \mathscr{T}(\mathscr{P})$ but not to $\mathscr{2} \mathscr{T}(\mathscr{P} \cup \mathscr{S})$. 
Hence, $\mathscr{2} \mathscr{T}(\mathscr{P} \cup \mathscr{S}) \varsubsetneqq \mathscr{Q T}(\mathscr{P})$. We conclude that $\mathscr{P}$ contains all but perhaps finitely many of the projections in $\mathscr{P} \cup \mathscr{S}$.

By symmetry, $\mathscr{S}$ contains all but perhaps finitely many of the projections in $\mathscr{P} \cup \mathscr{S}$. So there exists a positive integer $k$ such that $\left\{P_{n}: \operatorname{dim} P_{n} \geqq k\right\} \subseteq \mathscr{S}$ and $\left\{S_{n}: \operatorname{dim} S_{n} \geqq k\right\} \subseteq \mathscr{P}$. Let $m_{0}$ be the first positive integer such that $\operatorname{dim}\left(P_{m_{0}}\right) \geqq k$ and let $n_{0}$ be the first integer such that $\operatorname{dim}\left(S_{n_{0}}\right) \geqq k$. Then $P_{m_{0}+k}=S_{n_{0}+k}$ for all $k \in \mathbf{N}$.

THEOREM 10. $\mathscr{S}=\left\{S_{n}\right\}_{n=1}^{\infty}$ is a defining sequence for $\mathscr{Q T}(\mathscr{P})$ if and only if there exist positive integers $m_{0}$ and $n_{0}$ such that $\lim _{k}\left\|P_{m_{0}+k}-S_{n_{0}+k}\right\|=0$.

Proof. $\Leftarrow$ : We note that $\mathscr{2} \mathscr{T}(\mathscr{S}) \subseteq \mathscr{2} \mathscr{T}(\mathscr{P})$ since for $T$ in $\mathscr{Q T}(\mathscr{T})$,

$$
\begin{aligned}
& \left\|P_{m_{0}+k}^{\perp} T P_{m_{0}+k}\right\| \leqq\left\|S_{n_{0}+k}^{\perp} T S_{n_{1}+k}\right\|+\left\|\left(P_{m 0^{+}+k}^{\perp}-S_{n_{0}+k}^{\perp}\right) T S_{n_{0}+k}\right\| \\
& +\left\|P_{m 0^{+} k}^{\perp} T\left(P_{m_{0}+k}-S_{n_{0}+k}\right)\right\| \\
& \leqq\left\|S_{n_{0}+k}^{\perp} T S_{n 0^{+}+k}\right\|+\left\|P_{m 0^{+} k}^{\perp}-S_{n_{0+1}+k}^{\perp}\right\| \cdot\|T\| \\
& +\|T\| \cdot\left\|P_{m_{0}+k}-S_{n_{0}+k}\right\|
\end{aligned}
$$

and the other inclusion follows by symmetry.

$\Rightarrow$ : We assume that $\mathscr{S}=\left\{S_{n}\right\}_{n=1}^{\infty}$ is a defining sequence for $\mathscr{2} \mathscr{T}(\mathscr{P})$. Let $V$ be any unitary operator such that $\left\{V S_{n} V^{*}\right\}_{n=1}^{\infty} \cup\left\{P_{n}\right\}_{n=1}^{\infty}$ is a sequence of projections totally ordered by set inclusion.

Let $\mathscr{W}=\left\{W_{n}\right\}_{n=1}^{\infty}$ with $W_{n}=V S_{n} V^{*}$ for each $n$. We assert that $V$ belongs to $2 \mathscr{T}(\mathscr{W})$. So assume that $T$ is strictly upper triangular for $\mathscr{W}$; it suffices to show that $V T V^{*}$ belongs to $\mathscr{Q T}(\mathscr{W})$ by Remark 5 . By Remark 6, $T$ belongs to $2 \mathscr{T}(\mathscr{P} \cup \mathscr{W}) \subseteq 2 \mathscr{T}(\mathscr{P})$ so that it remains to observe that $V \mathscr{2} \mathscr{T}(\mathscr{P}) V^{*} \subseteq \mathscr{2} \mathscr{T}(\mathscr{W}): \quad W_{n}^{\perp}\left(V T V^{*}\right) W_{n}=$ $\left(V S_{n}^{\perp} V^{*}\right)\left(V T V^{*}\right)\left(V S_{n} V^{*}\right)=V S_{n}^{\perp} T S_{n} V^{*}$, so that $\left\|W_{n}^{\perp}\left(V T V^{*}\right) W_{n}\right\|=$ $\left\|V S_{n}^{\perp} T S_{n} V^{*}\right\|=\left\|S_{n}^{\perp} T S_{n}\right\| \rightarrow 0$.

Hence, we conclude that $V$ belongs to $\mathscr{2} \mathscr{T}(\mathscr{W})$. Since $2 \mathscr{T}(\mathscr{W})$ is inverse-closed by Lemma 2, it follows that $\left\|W_{n}^{\perp} V W_{n}\right\| \rightarrow 0$ and $\left\|W_{n} V W_{n}^{\perp}\right\|=\left\|W_{n}^{\perp} V^{*} W_{n}\right\| \rightarrow 0$.

(1) Since $W_{n} V=V S_{n}$, we have that $W_{n} V W_{n}^{\perp}=V S_{n} W_{n}^{\perp}$ so that $\left\|W_{n} V W_{n}^{\perp}\right\|=\left\|V S_{n} W_{n}^{\perp}\right\|=\left\|S_{n} W_{n}^{\perp}\right\| \rightarrow 0$ and

(2) Since $W_{n}^{\perp} V=V S_{n}^{\perp}$, we have that $W_{n}^{\perp} V W_{n}=V S_{n}^{\perp} W_{n}$ so that $\left\|W_{n}^{\perp} V W_{n}\right\|=\left\|V S_{n}^{\perp} W_{n}\right\|=\left\|S_{n}^{\perp} W_{n}\right\| \rightarrow 0$.

Since $\left\|S_{n}-W_{n}\right\|=\max \left\{\left\|S_{n}^{\perp} W_{n}\right\|,\left\|S_{n} W_{n}^{\perp}\right\|\right\}$ [5, Lemma 6] it follows that $\lim _{n}\left\|S_{n}-W_{n}\right\|=0$ and by a previous argument that $\mathscr{W}$ is a defining sequence for $\mathscr{Q} \mathscr{T}(\mathscr{P})$. It follows from Lemma 9 that there are integers $m_{0}$ and $n_{0}$ such that $W_{n_{0}+k}=P_{m_{0}+k}$ for all $k$ in $\mathbf{N}$. Hence 


$$
\lim _{k}\left\|S_{n_{0}+k}-P_{m_{0}+k}\right\|=0
$$

which concludes the proof.

EXAmPle 11. As an easy consequence of Theorem 10, it follows that there exist defining sequences $\mathscr{P}=\left\{P_{n}\right\}_{n=1}^{\infty}$ and $\mathscr{R}=\left\{R_{n}\right\}_{n=1}^{\infty}$ for a quasitriangular algebra $\mathscr{A}$ such that $\left\{P_{n} \vee R_{n}\right\}_{n=1}^{\infty}$ is not a defining sequence for $\mathscr{A}$ (" $v$ " denotes the supremum of two projections). This phenomenon is suggested by an example in [3, p. 285].

We shall say that two subsets of $\mathscr{L}(\mathscr{H}), \mathscr{S}$ and $\mathscr{T}$, are locally isomorphic if each operator in $\mathscr{S}$ is unitarily equivalent to an operator in $\mathscr{T}$ and conversely. Because every quasitriangular operator is a compact perturbation of a triangular operator, it follows that any two quasitriangular algebras are locally isomorphic; from Theorem 12 we conclude that they are not necessarily isomorphic.

THEOREM 12. Let $\mathscr{2} \mathscr{T}(\mathscr{P})$ and $\mathscr{2 T}(\mathscr{T})$ be quasitriangular algebras. Then $\mathscr{2} \mathscr{T}(\mathscr{P})$ and $\mathscr{2} \mathscr{T}(\mathscr{S})$ are algebraically isomorphic if and only if there exist positive integers $j_{0}$ and $l_{0}$ such that $\operatorname{dim}\left(P_{j_{0}+k}\right)=\operatorname{dim}\left(S_{k_{0}+k}\right)$ for all $k$ in N.

Proof. $\Leftarrow$ : If we assume that there exist positive integers $j_{0}$ and $l_{0}$ such that $\operatorname{dim}\left(P_{j_{0}+k}\right)=\operatorname{dim}\left(S_{l_{0}+k}\right)$ for all $k$ in $\mathbf{N}$, then we can define a unitary operator $U$ such that $U P_{j 0^{+} k} U^{*}=S_{k+k}$ for all $k$ in $\mathbf{N}$. We assert that $U$ implements an isomorphism from $\mathscr{Q T}(\mathscr{P})$ to $\mathscr{2} \mathscr{T}(\mathscr{Y})$.

$\Rightarrow$ : Assume that there is a map $\alpha$ from $2 \mathscr{T}(\mathscr{P})$ to $\mathscr{2} \mathscr{T}(\mathscr{S})$ which preserves algebraic structure. Since $\mathscr{2} \mathscr{T}(\mathscr{P})$ and $\mathscr{2} \mathscr{T}(\mathscr{S})$ are Banach algebras, each of which contains the set of finite rank operators, it follows from [6, Theorem 2.5.19] that there exists an invertible operator $S$ such that $\alpha(T)=S T S^{-1}$ for all $T$ in $\mathscr{Q T}(\mathscr{P})$.

We conlude from [1, Theorem 3.3] that $S$ has a factorization $S=U A$ where $A$ belongs to $\mathscr{T}(\mathscr{P})$ and $U$ is unitary. Then we note that $R_{n}=U P_{n} U^{*}$ is a defining sequence for $\mathscr{2} \mathscr{T}(\mathscr{T})$; by Theorem 10 , we note that there exist positive integers $m_{0}$ and $n_{0}$ such that $\left\|R_{m_{0+k}}-S_{n^{+}+k}\right\| \rightarrow 0$. So, there exists a positive integer $d$ such that $\left\|R_{m_{0}+d+k}-S_{n_{0}+d+k}\right\|<1$ for all $k$ in $\mathbf{N}$. Hence, $\operatorname{dim}\left(R_{m_{0}+d+k}\right)=\operatorname{dim}\left(S_{n_{0}+d+k}\right)$ for all $k$ in $\mathbf{N}$. Since $\operatorname{dim}\left(P_{n}\right)=\operatorname{dim}\left(R_{n}\right)$ for all $n$ in $\mathbf{N}$, let $j_{0}=m_{0}+d$ and let $l_{0}=n_{0}+d$ to obtain the theorem.

\section{REFERENCES}

1. W. B. Arveson, Interpolation problems in nest algebras, J. Functional Analysis, 20 (1975), $208-233$. 
2. R. G. Douglas and C. Pearcy, A note on quasitriangular operators, Duke Math. J., 37 (1970), $177-188$.

3. P. R. Halmos, Quasitriangular operators, Acta Sci. Mat. (Szeged), 29 (1968), 283-293.

4. — Ten problems in Hilbert space, Bulletin Amer. Math. Soc., 76 (1970), 887-933.

5. J. K. Plastiras, Compact perturbations of certain von Neumann algebras, Trans. Amer. Math. Soc., (to appear).

6. C. E. Rickart, General Theory of Banach Algebras, D. van Nostrand Company, Inc. (1960).

7. B. Sz.-Nagy, Specktraldarstellung linearer Transformation des Hilbertschen Raumes, Berlin, (1942).

Received February 23, 1976.

University of Pennsylvania 



\section{PACIFIC JOURNAL OF MATHEMATICS}

\section{EDITORS}

RICHARD ARENS (Managing Editor)

University of California

Los Angeles, California 90024

\author{
R. A. Beaumont \\ University of Washington \\ Seattle, Washington 98105
}

\section{J. DugunduI}

Department of Mathematics University of Southern California Los Angeles, California 90007

D. Gilbarg and J. Milgram

Stanford University

Stanford, California 94305

\section{ASSOCIATE EDITORS}

E. F. BECKENBACH
B. H. NeumanN

F. Wolp
K. YoshidA

\section{SUPPORTING INSTITUTIONS}

UNIVERSITY OF BRITISH COLUMBIA

CALIFORNIA INSTITUTE OF TECHNOLOGY

UNIVERSITY OF CALIFORNIA

MONTANA STATE UNIVERSITY

UNIVERSITY OF NEVADA

NEW MEXICO STATE UNIVERSITY

OREGON STATE UNIVERSITY

UNIVERSITY OF OREGON

OSAKA UNIVERSITY

\author{
UNIVERSITY OF SOUTHERN CALIFORNIA \\ STANFORD UNIVERSITY \\ UNIVERSITY OF HAWAII \\ UNIVERSITY OF TOKYO \\ UNIVERSITY OF UTAH \\ WASHINGTON STATE UNIVERSITY \\ UNIVERSITY OF WASHINGTON \\ AMERICAN MATHEMATICAL SOCIETY
}

The Supporting Institutions listed above contribute to the cost of publication of this Journal, but they are not owners or publishers and have no responsibility for its contents or policies.

Mathematical papers intended for publication in the Pacific Journal of Mathematics should be in typed form or offset-reproduced (not dittoed). double spaced with large margins. Underline Greek letters in red, German in green, and script in blue. The first paragraph or two must be capable of being used separately as a synopsis of the entire paper. Items of the biblography should not be cited there unless absolutely necessary, in which case they must be identified by author and Journal, rather than by item number. Manuscripts, in duplicate, may be sent to any one of the four editors. Please classify according to the scheme of Math. Reviews, Index to Vol. 39. All other communications should be addressed to the managing editor, or Elaine Barth, University of California, Los Angeles, California, 90024.

100 reprints are provided free for each article, only if page charges have been substantially paid. Additional copies may be obtained at cost in multiples of 50 .

The Pacific Journal of Mathematics is issued monthly as of January 1966. Regular subscription rate: $\$ 72.00$ a year (6 Vols., 12 issues). Special rate: $\$ 36.00$ a year to individual members of supporting institutions.

Subscriptions, orders for back numbers, and changes of address should be sent to Pacific Journal of Mathematics, 103 Highland Boulevard, Berkeley, California, 94708.

PUBLISHED BY PACIFIC JOURNAL OF MATHEMATICS, A NON-PROFIT CORPORATION Printed at Jerusalem Academic Press, POB 2390, Jerusalem, Israel.

Copyright (C) 1976 Pacific Journal of Mathematics All Rights Reserved 


\section{Pacific Journal of Mathematics}

\section{Vol. 64, No. $2 \quad$ June, 1976}

Richard Fairbanks Arnold and A. P. Morse, Plus and times............. 297

Edwin Ogilvie Buchman and F. A. Valentine, External visibility ......... 333

R. A. Czerwinski, Bonded quadratic division algebras.............. 341

William Richard Emerson, Averaging strongly subadditive set functions in unimodular amenable groups. II .........................

Lynn Harry Erbe, Existence of oscillatory solutions and asymptotic behavior for a class of third order linear differential equations ............. 369

Kenneth R. Goodearl, Power-cancellation of groups and modules........ 387

J. C. Hankins and Roy Martin Rakestraw, The extremal structure of locally compact convex sets ...................................

Burrell Washington Helton, The solution of a Stieltjes-Volterra integral

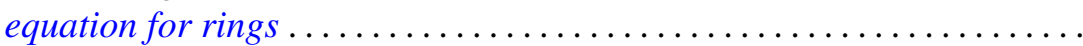

Frank Kwang-Ming Hwang and Shen Lin, Construction of 2-balanced

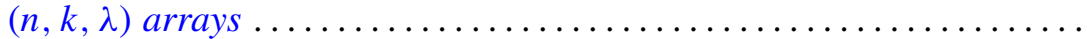

Wei-Eihn Kuan, Some results on normality of a graded ring ... 455

Dieter Landers and Lothar Rogge, Relations between convergence of series and convergence of sequences ......................... 465

Lawrence Louis Larmore and Robert David Rigdon, Enumerating immersions and embeddings of projective spaces ................

Douglas C. McMahon, On the role of an abelian phase group in relativized problems in topological dynamics..................

Robert Wilmer Miller, Finitely generated projective modules and TTF classes...

Yashaswini Deval Mittal, A class of isotropic covariance functions ...

Anthony G. Mucci, Another martingale convergence theorem ...

Joan Kathryn Plastiras, Quasitriangular operator algebras ...

John Robert Quine, Jr., The geometry of $p\left(S^{1}\right) \ldots \ldots \ldots$. 